Research Article

\title{
Distributed Finite-Time Bipartite Consensus for Multiagent System with Event-Triggered Control
}

\author{
Haibo Xie $\mathbb{i}$, Zhengjiang Liu, Chengyong Yan, and Shibo Zhou \\ Navigation College, Dalian Maritime University, Dalian 116026, China \\ Correspondence should be addressed to Haibo Xie; xiehaibo@dlmu.edu.cn
}

Received 6 January 2020; Revised 1 May 2020; Accepted 10 July 2020; Published 27 July 2020

Academic Editor: Quanxin Zhu

Copyright (c) 2020 Haibo Xie et al. This is an open access article distributed under the Creative Commons Attribution License, which permits unrestricted use, distribution, and reproduction in any medium, provided the original work is properly cited.

\begin{abstract}
This paper investigates the distributed finite-time event-triggered bipartite consensus control for multiagent systems over antagonistic networks. Under the constraint of energy conservation, a distributed nonlinear finite-time control protocol only depending upon local information is proposed coupled with event-triggered strategies, where controllers of agents at triggered instants are only updated to reduce the computation. It is proved that when the antagonistic network is structurally balanced with a spanning tree, a necessary and sufficient condition is established to guarantee all agents to reach consensus values with identical magnitude but opposite signs. More interestingly, the settling time depending on the initial state is obtained over the whole process. Comparing to asymptotic control algorithms, the proposed control method has better disturbance rejection properties and convergence rate. Simulations are given to demonstrate the effectiveness of the theoretical results.
\end{abstract}

\section{Introduction}

Recently, coordination control for multiagent systems (MASs) has received enormous attention due to its widespread applications, such as marine environment monitoring [1], multiships cooperative combat [2], and source localization $[3,4]$. Depending on the different theories, these coordination control problems are mainly classified into three types: consensus [5-7], flocking [8-10], and formation control $[11,12]$. Notably, the consensus is regarded as a fundamental coordination problem, which indicates that the states of all agents asymptotically achieve the same value. Over a directed graph, Hua et al. [7] have addressed the leader-following output consensus for a class of nonlinear MASs with time delay. As a common feature in existing solutions, the cooperative interactions between agents were typically modeled by a communication topology associated with nonnegative edge weights. However, communication topologies with antagonistic interactions containing both positive and negative edge weights are common, especially in the social network theories [13-15]. This communication topology is called a signed network [16]. Subsequently, the real challenge to reach consensus is generated for MASs over a signed network. Meanwhile, a notion of bipartite consensus is proposed with the assistance of signed network theory $[13,14]$, where all agents converge to values with the same magnitude but opposite signs.

Ideally, for bipartite consensus control of MASs, one of the key elements is to have a continuous measurement with an embedded sensor to take control actions, i.e., the agent continuously updates its controller via measuring its neighbors' state all the time [17, 18]. However, as the number of agents increased, the capacities of communication and computation are strictly limited. Therefore, it is extremely significant to design a reasonable mechanism for information sharing and controller updating. To this end, a sampleddata control method was applied for the bipartite consensus problem of MASs [19], in which the measurements are taken periodically, as well as the control actions synchronously. However, this method could lead to excessive consumption of communication and computation. In order to utilize resources more effectively, an event-triggered strategy was proposed consequently in $[20,21]$, where an event-triggered condition is firstly constructed, and then the controller is only updated when the event-triggered condition is satisfied. Furthermore, according to a dynamic rule designed, Li et al. 
[22] investigated the dynamically adjusted threshold parameter in the improved event-triggered condition. Additionally, by taking MASs with input saturation into consideration, $\mathrm{Xu}$ et al. [23] developed an event-triggered control mechanism coupled with the low-gain feedback technique.

On the contrary, the convergence time is a significant performance indicator for a bipartite consensus protocol. In most existing works, over an infinite time horizon, these MASs can exponentially achieve values with the same magnitude but opposite signs on states. Generally, a pioneering work in $[13,14,24]$ reveals that the convergence time is determined by the second smallest eigenvalue of the graph Laplacian. Nevertheless, the finite-time bipartite consensus is more desirable for specific system requirements, e.g., multiships accurately hunt a military vessel in finite time. Thus, finite-time bipartite consensus has elicited some researchers' attention [25-27]. Under the structurally balanced signed graph, Meng et al. [26] have investigated the finite-time bipartite consensus control for nonlinear systems. Furthermore, with regards to the case of second-order MASs with antagonistic interactions and unknown external disturbances, Zhao et al. [27] have addressed the adaptive finite-time bipartite consensus problems. However, to our best knowledge, less attention has been given to the distributed finite-time event-triggered bipartite consensus. In this paper, different from considering the event-driven control for finite-time consensus in the existing work $[24,25]$, we aim at achieving distributed finite-time bipartite consensus for MASs by using event-triggered strategies. The main contributions of this survey are the following. First, distinct from the most previous event-triggered strategy based on a linear controller, an improved finite-time nonlinear controller is designed over signed network coupled with the event-triggered strategy. When the signed network is structurally balanced with a spanning tree, the distributed finite-time bipartite consensus is implemented using local information. Second, the event-triggered condition is built using Lyapunov stability theory. In subsequent simulation, the reductions in number of controller update are attained with superior performance. Finally, depending on initial states of agents, the upper bound of convergence time is obtained during the whole process.

The remainder of this paper is organized as follows. Preliminary definitions and the problem formulation are presented in Section 2. The main results are described in Section 3. Section 4 discusses the simulation examples before we conclude in Section 5.

\section{Preliminaries and Problem Statement}

In this section, we first define related notions and collect basic concepts from algebraic graph theory, which will be used throughout this paper. Then, the concerned system model and bipartite consensus problem are formulated.

2.1. Preliminaries. $\mathbb{R}$ represents the set of real numbers. $\mathbb{R}^{N \times N}$ denotes a $N \times N$ real matrix. $I_{N}$ is $N$ dimension unit matrix. $\mathbf{1}_{N}$ and $\mathbf{0}_{N}$ stand for $N$ dimension column vectors with all entries 1 and 0 , respectively. $|S|$ is the absolute value of each element of the vector or matrix $S$. The matrix $\operatorname{diag}\left(\lambda_{1}, \lambda_{2}, \ldots, \lambda_{N}\right)$ denotes a diagonal matrix with diagonal entries $\lambda_{1}, \lambda_{2}, \ldots, \lambda_{N}$.

The interaction network between agents is described by an undirected signed graph $\mathscr{G}, \mathscr{G}=(v, \mathscr{E}, \mathscr{A})$, where $v=$ $\left\{v_{1}, v_{2}, \ldots, v_{N}\right\}$ denotes a set of nodes, $\mathscr{E}=v \times v$ represents a set of edges, and $\mathscr{A}=\left[a_{i j}\right] \in \mathbb{R}^{N \times N}$ is a matrix of the signed weights. In $\mathscr{A}, a_{i j} \neq 0$ is well established if and only if $\left(v_{j}, v_{i}\right) \in \mathscr{E}$, otherwise $a_{i j}=0$. Note that graphs with selfloops $a_{i i}=0,(i=1,2, \ldots, N)$ is not taken into consideration. For the signed graph $\mathscr{G}$, the edge set $\mathscr{E}=\mathscr{E}^{+} \cup \mathscr{E}^{-}$is denoted by $\mathscr{E}^{+}=\left\{\left(v_{j}, v_{i}\right) \mid a_{i j}>0\right\} \quad$ and $\mathscr{E}^{-}=\left\{\left(v_{j}, v_{i}\right) \mid a_{i j}<0\right\}$. Moreover, there exists $\left(v_{j}, v_{i}\right) \in \mathscr{E}$ such that agent $j$ is called a neighbor of agent $i$. $\mathcal{N}_{i}=\left\{\left(v_{j}, v_{i}\right) \in \mathscr{E}, j \neq \mathrm{i}\right\}$ is used to represent a neighbor set of agent $i$. Note that irreducibility of $\mathscr{A}$ corresponds to $\mathscr{G}$ which is strongly connected, i.e., a path from node $v_{i}$ to node $v_{j}$ is a finite sequence of edges in the form of $\left(v_{i}, v_{i_{k 1}}\right),\left(v_{i_{k 1}}, v_{i_{k 2}}\right), \ldots,\left(v_{i_{k l}}, v_{j}\right), i \neq j$.

For a signed graph $\mathscr{G}$, the corresponding Laplacian matrix $L$ is defined as

$$
L=\operatorname{diag}\left(\sum_{k \in \mathcal{N}_{1}}\left|a_{1 k}\right|, \sum_{k \in \mathcal{N}_{2}}\left|a_{2 k}\right|, \ldots, \sum_{k \in \mathcal{N}_{N}}\left|a_{N k}\right|\right)-\mathscr{A} \text {. }
$$

Then, the eigenvalues of $L$ can be indicated by a decreasing order:

$$
\lambda_{N}(L) \geq \cdots \geq \lambda_{2}(L)>\lambda_{1}(L)=0 .
$$

Given any signed graph $\mathscr{G}$, there exists a bipartition with $v_{1}$ and $v_{2}$, which satisfy condition $v_{1} \cup v_{2}=v$, and $v_{1} \cap v_{2}=\varnothing$. When the signed weight is $a_{i j} \geq 0$ for $\forall v_{i}, v_{j} \in v_{q}(q \in\{1,2\}), \quad$ and $a_{i j} \leq 0$ for $\forall v_{i} \in v_{q}, v_{j} \in v_{r}, q \neq r(q, r \in\{1,2\}), \mathscr{G}$ can be regarded as structurally balanced, otherwise structurally unbalanced.

To facilitate subsequent proof and analysis, the following lemmas are introduced.

Lemma 1 (see [13]). For a structurally balanced signed graph $\mathscr{G} \mathscr{G}$, there exists a diagonal matrix $D=\operatorname{diag}\left\{\sigma_{1}, \sigma_{2}, \ldots, \sigma_{N}\right\}$ such that the entries of $D \mathscr{A} D$ are all nonnegative, where $\sigma_{i} \in\{1,-1\}, \forall i \in v$.

Lemma 2 (see [28]). Given a non-Lipschitz continuous nonlinear system $\dot{x}(t, x(0))=g(x)$, there exists a continuous function $W(x)$ defined on a neighborhood of the origin, called the settling-time function, such that the following conditions hold:

(1) $W(x)$ is positive definite.

(2) $\dot{W}(x)+b W^{\gamma}(x) \leq 0, \quad b>0,0<\gamma<1$.

Then, the origin is locally finite-time stable if (i) system $\dot{x}(t, x(0))=g(x)$ is stable in an open neighborhood of the origin and (ii) there exists $0<W(x(0))<\infty$ for which 
$x(t, x(0))=0$ for all $t>W(x(0))$. Moreover, the settling time $T=\inf \{W(x(0)) \geq 0: x(t, x(0))=0 \forall t \geq W(x(0))\}$ satisfies

$$
T \leq \frac{1}{b(1-\gamma)} W(x(0))^{b(1-\gamma)},
$$

for all $x(0)$ in some open neighborhood of the origin.

Lemma 3 (see $\quad[29]) . \quad\left(d|x|^{1+\gamma}\right) /(d t)=((1+\gamma)$ $\left.\operatorname{sgn}(x)|x|^{\gamma} d x\right) /(d t)$, where $x \in \mathbb{R}, \gamma \in \mathbb{R}$ and the sign function $\operatorname{sgn}(\cdot)$ is described as

$$
\operatorname{sgn}(x)= \begin{cases}1, & x>0 \\ 0, & x=0 \\ -1, & x<0\end{cases}
$$

Lemma 4. Given any $y_{1}, y_{2}, \ldots, y_{N} \geq 0$ and $0<p \leq 1$, the following properties are applied:

$$
\sum_{i=1}^{N} y_{i}^{p} \geq\left(\sum_{i=1}^{N} y_{i}\right)^{p} .
$$

2.2. Problem Formulation. For a signed graph $\mathscr{G}$, considering a group of $N$ single-integrator agents, which can be modeled as follows:

$$
\dot{x}_{i}(t)=u_{i}(t), \quad i \in v,
$$

where $x_{i}(t) \in \mathbb{R}^{n}$ and $u_{i}(t) \in \mathbb{R}^{n}$ are the state and the control input of agent $i$, respectively. For simplicity, we employ $n=1$ in next analysis.

Suppose that the $N$ agents are classified into two antagonistic groups $v_{1}$ and $v_{2}$, where $v_{1} \cup v_{2}=v$ and $v_{1} \cap v_{2}=\varnothing$. Then, an interaction network of all agents can be described with a structurally balanced signed graph $\mathscr{G}$.

Compared with the existing asymptotic bipartite consensus schemes over signed graph, the finite-time bipartite consensus is more desirable due to its better performance in higher precision, better robustness, and faster convergence rate [28].

The definition of finite-time bipartite consensus for MASs is described as follows.

Definition 1. (finite-time bipartite consensus). Given a structurally balanced signed graph $\mathscr{G}$, a distributed control law $u_{i}\left(t, x_{i}(t)\right), i=1,2, \ldots, N$, is designed to achieve the finite-time bipartite consensus for system (7). Namely, there exists a settling time $T$ such that

$$
\begin{aligned}
\lim _{t \longrightarrow T} x_{i}(t)=\sigma_{i} \varepsilon, & \forall i \in v_{1}, \\
\lim _{t \longrightarrow T} x_{j}(t)=\sigma_{j} \varepsilon, & \forall j \in v_{2}, \\
\left|x_{i}\right|=\left|x_{j}\right|, & \text { if } t>T,
\end{aligned}
$$

where $\sigma_{i}, \sigma_{j} \in\{1,-1\}$ is defined in Lemma 1 and $\varepsilon=(1 / N)\left(\mathbf{1}_{N}^{T} x(0)\right) \mathbf{1}_{N}$ is the same absolute value of the final states of all agents.

In the networks with antagonistic links, a continuous nonlinear finite-time bipartite consensus protocol is designed as

$$
\begin{aligned}
u_{i}(t)= & \beta \sum_{j \in \mathcal{N}_{i}} a_{i j} \operatorname{sgn}\left(x_{j}(t)-\operatorname{sgn}\left(a_{i j}\right) x_{i}(t)\right) \\
& \cdot\left\|x_{j}(t)-\operatorname{sgn}\left(a_{i j}\right) x_{i}(t)\right\|^{\alpha},
\end{aligned}
$$

where $\alpha \in(0,1), \beta>0$, and $\operatorname{sgn}(\cdot)$ is the sign function.

Note that the control protocol (9) needs to be continuously updated, which causes to an undesirable consumption of communication and computation. To address this problem, an event-triggered strategy is applied to the avoidance of undesirable consumption. To be particular, in the scenarios of continuous communication, the control actions are only taken at discrete event instants determined by event-triggered condition, which indicate that between two event-triggered sampling instants, the controllers are regarded as zero-order holder.

Denote an increasing sequence $t_{0}^{i}, t_{1}^{i}, \ldots, t_{k}^{i}, \ldots$ as the event instants of agent $i$, such that $\hat{x}_{i}(t)=x_{i}\left(t_{k}^{i}\right)$ is the state of agent $i$ at the $k$ th event instants. It is noteworthy that $x_{i}(t)$ at $t \in\left[t_{k}^{i}, t_{k+1}^{i}\right)$ may receive several broadcasted information from its neighbor $j$. However, agent $i$ will not update control action immediately upon receival. Namely, the controller of agent $i$ at $t \in\left[t_{k}^{i}, t_{k+1}^{i}\right)$ could be regarded as the zero-order holder. From (9), a distributed nonlinear finite-time bipartite consensus control protocol is proposed coupled with the event-triggered mechanism:

$$
\begin{aligned}
u_{i}(t)= & \beta \sum_{j \in \mathcal{N}_{i}} a_{i j} \operatorname{sgn}\left(x_{j}\left(t_{k}^{j}\right)-\operatorname{sgn}\left(a_{i j}\right) x_{i}\left(t_{k}^{i}\right)\right) \\
& \cdot\left\|x_{j}\left(t_{k}^{j}\right)-\operatorname{sgn}\left(a_{i j}\right) x_{i}\left(t_{k}^{i}\right)\right\|^{\alpha},
\end{aligned}
$$

where $k \prime=\operatorname{argmin}_{l \in N_{+}: t \geq t^{j}}\left(t-t_{l}^{j}\right)$.

For simplicity, let the state measurement error for agent $i$ be $\hat{x}_{i}(t)$ :

$$
e_{i}(t)=\widehat{x}_{i}(t)-x_{i}(t)
$$

Then, replacing (11) into (10), (10) is rewritten as

$$
\begin{aligned}
u_{i}(t) & =\beta \sum_{j \in \mathcal{N}_{i}} a_{i j} \operatorname{sgn}\left(x_{j}(t)+e_{j}(t)-\operatorname{sgn}\left(a_{i j}\right)\left(x_{i}(t)+e_{i}(t)\right)\right)\left\|\left(x_{j}(t)+e_{j}(t)-\operatorname{sgn}\left(a_{i j}\right)\left(x_{i}(t)+e_{i}(t)\right)\right)\right\|^{\alpha} \\
& =\beta \sum_{j \in \mathcal{N}_{i}} a_{i j} \operatorname{sgn}\left(\left(x_{j}(t)-\operatorname{sgn}\left(a_{i j}\right) x_{i}(t)\right)+\left(e_{j}(t)-\operatorname{sgn}\left(a_{i j}\right) e_{i}(t)\right)\right)\left\|\left(x_{j}(t)-\operatorname{sgn}\left(a_{i j}\right) x_{i}(t)\right)+\left(e_{j}(t)-\operatorname{sgn}\left(a_{i j}\right) e_{i}(t)\right)\right\|^{\alpha} .
\end{aligned}
$$


Let

$$
\begin{aligned}
& \delta_{i}(t)=\sum_{j \in \mathcal{N}_{i}} a_{i j}\left(x_{j}(t)-\operatorname{sgn}\left(a_{i j}\right) x_{i}(t)\right), \\
& E_{i}(t)=\sum_{j \in \mathcal{N}_{i}} a_{i j}\left(e_{j}(t)-\operatorname{sgn}\left(a_{i j}\right) e_{i}(t)\right) .
\end{aligned}
$$

Substituting (13) into system (12), the closed-loop form of agent $i$ is presented:

$$
\dot{x}_{i}(t)=\beta \operatorname{sgn}\left(\delta_{i}(t)+E_{i}(t)\right)\left\|\delta_{i}(t)+E_{i}(t)\right\|^{\alpha} .
$$

For system (7), the following questions will be addressed. (1) How to design the event-triggered condition to determine the sampling instant $t_{k}^{i}$ ? (2) Under the event-triggered condition designed, can the distributed event-triggered bipartite consensus be solvable in finite time?

\section{Main Result}

In this section, we will answer all the questions raised at the end of the previous section and provide the main results and theoretical analyses.

Theorem 1. Considering a MASs (7) and the control protocol (10) under a structurally balanced signed graph $\mathscr{G}$, the finitetime bipartite consensus is achieved in settling time $T$ satisfying

$$
T=\frac{(1+\alpha) V(0)^{(1-\alpha) /(1+\alpha)}}{c^{\prime}(1-\alpha)}
$$

when the event-triggered condition is designed as

$$
\left\|E_{i}(t)\right\| \leq c_{i}\left\|\delta_{i}(t)\right\|
$$

where $\alpha \in(0,1), \quad V(0)=\sum_{i=1}^{N}(\beta /(1+\alpha))\left\|\delta_{i}(0)\right\|^{1+\alpha}$ is the Lyapunov function at initial instant $t=0$, $c^{\prime}=\left(\left(c \beta^{2}\right) /\left((\beta /(1+\alpha))^{((2 \alpha) /(1+\alpha))}\right)\right), \beta>0$, and $c_{i}$ is a positive scalar.

Proof. Considering a candidate Lyapunov function,

$$
V(t)=\sum_{i=1}^{N} \frac{\beta}{1+\alpha}\left\|\delta_{i}(t)\right\|^{1+\alpha} \text {. }
$$

Evidently, one obtains that $V(t)>0$ and $V(t)=0$ when the finite-time bipartite consensus is achieved. Using Lemma 3, the derivative of the Lyapunov function (17) along the trajectories of system (7) leads to

$$
\dot{V}(t)=\sum_{i=1}^{N} \frac{\beta}{1+\alpha} \frac{\mathrm{d}\left\|\delta_{i}(t)\right\|^{1+\alpha}}{\mathrm{d} t}=\sum_{i=1}^{n} \beta \operatorname{sgn}\left(\delta_{i}\right)\left\|\delta_{i}(t)\right\|^{\alpha} \dot{\delta}_{i}(t) .
$$

Together with the definition of (13) and control protocol (10), we obtain

$$
\begin{aligned}
\dot{\delta}_{i}(t) & =\sum_{j \in \mathcal{N}_{i}} a_{i j}\left(\dot{\delta}_{j}(t)-\operatorname{sgn}\left(a_{i j}\right) \dot{\delta}_{i}(t)\right) \\
& =\sum_{j \in \mathcal{N}_{i}} a_{i j}\left(\dot{x}_{j}(t)-\operatorname{sgn}\left(a_{i j}\right) \dot{x}_{i}(t)\right) \\
& =-\sum_{j \in \mathcal{N}_{i}}\left(a_{i j} \operatorname{sgn}\left(a_{i j}\right) \dot{x}_{i}(t)+a_{i j} \dot{x}_{j}(t)\right) \\
& =-\sum_{j \in \mathcal{N}_{i}} l_{i j} \dot{x}_{j}(t) \\
& =-\sum_{j \in \mathcal{N}_{i}} l_{i j} \beta \operatorname{sgn}\left(\delta_{j}(t)+E_{j}(t)\right)\left\|\delta_{j}(t)+E_{j}(t)\right\|^{\alpha}
\end{aligned}
$$

where $l_{i j}$ denotes $i$-row $j$-column element of Laplacian matrix $L$. Then, substituting (19) into (18), (18) can be rearranged as

$$
\begin{aligned}
\dot{V}(t) & =\sum_{i=1}^{N} \beta \operatorname{sgn}\left(\delta_{i}\right)\left\|\delta_{i}(t)\right\|^{\alpha}\left(-\sum_{j \in \mathcal{N}_{i}} l_{i j} \beta \operatorname{sgn}\left(\delta_{j}(t)+E_{j}(t)\right)\left\|\delta_{j}(t)+E_{j}(t)\right\|^{\alpha}\right) \\
& =-\sum_{i=1}^{N} \sum_{j \in \mathcal{N}_{i}}^{N}\left(\beta \operatorname{sgn}\left(\delta_{i}\right)\left\|\delta_{i}(t)\right\|^{\alpha}\right) l_{i j}\left(\beta \operatorname{sgn}\left(\delta_{j}(t)+E_{j}(t)\right)\left\|\delta_{j}(t)+E_{j}(t)\right\|^{\alpha}\right) .
\end{aligned}
$$

It is clear that

$$
\begin{gathered}
\operatorname{sgn}\left(\delta_{i}\right)\left\|\delta_{i}(t)\right\|^{\alpha} \leq\left\|\delta_{i}(t)\right\|^{\alpha} \\
\operatorname{sgn}\left(\delta_{j}(t)+E_{j}(t)\right)\left\|\delta_{j}(t)+E_{j}(t)\right\|^{\alpha} \leq\left\|\delta_{j}(t)+E_{j}(t)\right\|^{\alpha} \\
\leq\left(\left\|\delta_{j}(t)\right\|+\left\|E_{j}(t)\right\|\right)^{\alpha} \leq\left\|\delta_{j}(t)\right\|^{\alpha}+\left\|E_{j}(t)\right\|^{\alpha} .
\end{gathered}
$$

Replacing (21) and (22) into (20), it implies that

$$
\begin{array}{r}
\sum_{i=1}^{N} \sum_{j \in \mathscr{N}_{i}}^{N}\left(\beta \operatorname{sgn}\left(\delta_{i}\right)\left|\delta_{i}(t)\right|^{\alpha}\right) l_{i j}\left(\beta \operatorname{sgn}\left(\delta_{j}(t)+E_{j}(t)\right)\left\|\delta_{j}(t)+E_{j}(t)\right\|^{\alpha}\right) \\
\geq \sum_{i=1}^{N} \sum_{j \in \mathcal{N}_{i}}^{N}\left(\beta\left|\delta_{i}(t)\right|^{\alpha}\right) l_{i j}\left(\beta\left(\left\|\delta_{j}(t)\right\|^{\alpha}+\left\|E_{j}(t)\right\|^{\alpha}\right)\right) .
\end{array}
$$


Consequently, we can conclude that

$$
\begin{aligned}
V(t) & \leq-\sum_{i=1}^{N} \sum_{j \in \mathcal{N}_{i}}^{N}\left(\beta\left\|\delta_{i}(t)\right\|^{\alpha}\right) l_{i j}\left(\beta\left(\left\|\delta_{j}(t)\right\|^{\alpha}+\left\|E_{j}(t)\right\|^{\alpha}\right)\right) \\
& \leq-\sum_{i=1}^{N} \sum_{j \in \mathcal{N}_{i}}^{N}\left(\beta\left\|\delta_{i}(t)\right\|^{\alpha}\right) l_{i j}\left(\beta\left(\left(1+c_{j}^{\alpha}\right)\left\|\delta_{j}(t)\right\|^{\alpha}\right)\right) \\
& =-\left(\beta|\delta|^{\alpha}\right)^{T} L(I+C)\left(\beta|\delta|^{\alpha}\right) \\
& =-\left(\beta|\delta|^{\alpha}\right)^{T} Q\left(\beta|\delta|^{\alpha}\right),
\end{aligned}
$$

where $Q=L(I+C)$ and $C=\operatorname{diag}\left(c_{1}^{\alpha}, c_{2}^{\alpha}, \ldots, c_{N}^{\alpha}\right)$.

Define $\quad R=(1 / 2)\left(Q+Q^{T}\right)$ and $\Lambda=\left\{\theta \in \mathbb{R}: \theta^{T} \theta=\right.$ 1 and $\theta=\beta|\vartheta|^{\alpha}$ for some $\left.\vartheta \perp \mathbf{1}_{N}\right\}$. Here, $\Lambda$ is treated as a bounded closed set. For $\theta \in \Lambda$, the function $\theta^{T} R \theta$ is continuous with $\theta^{T} R \theta \neq 0$. Thus, let $\min _{\theta \in \Lambda} \theta^{T} R \theta$ be defined by $c$ with $c>0$. From $\mathbf{1}_{N} \perp \delta$, it yields that

$$
\frac{\left(\beta|\delta|^{\alpha}\right)^{T} Q\left(\beta|\delta|^{\alpha}\right)}{\left(\beta|\delta|^{\alpha}\right)^{T}\left(\beta|\delta|^{\alpha}\right)} \geq \min _{\theta \in \Lambda} \theta^{T} R \theta=c>0 .
$$

Assume that $\Gamma(t)=(\dot{V}(t)) /\left(V(t)^{(2 \alpha) /(1+\alpha)}\right)$, then

$$
\begin{aligned}
\Gamma(t) & \geq \frac{c\left(\beta|\delta|^{\alpha}\right)^{T}\left(\beta|\delta|^{\alpha}\right)}{V(t)^{(2 \alpha) /(1+\alpha)}} \\
& \geq \frac{c\left(\beta|\delta|^{\alpha}\right)^{T}\left(\beta|\delta|^{\alpha}\right)}{\left(\sum_{i=1}^{n}(\beta /(1+\alpha))\left|\delta_{i}(t)\right|^{1+\alpha}\right)^{(2 \alpha) /(1+\alpha)}} \\
& \geq \frac{c \sum_{i=1}^{n} \beta^{2}\left\|\delta_{i}(t)\right\|^{2 \alpha}}{\sum_{i=1}^{n}(\beta /(1+\alpha))^{(2 \alpha) /(1+\alpha)}\left\|\delta_{i}(t)\right\|^{2 \alpha}} .
\end{aligned}
$$

The last inequality can be obtained by Lemma 4 . For simplicity, let $c^{\prime}=\left(c \beta^{2}\right) /\left((\beta /(1+\alpha))^{(2 \alpha) /(1+\alpha)}\right)$, and we have

$$
\dot{V}(t) \leq-c^{\prime} V(t)^{(2 \alpha) /(1+\alpha)} \text {. }
$$

By Lemma 2, as $\delta(t)$ would be zero in finite time $T=\left((1+\alpha) V(0)^{(1-\alpha) /(1+\alpha)}\right) /\left(c^{\prime}(1-\alpha)\right)$, system (7) will achieve a bipartite consensus.

Remark 1. Here, the simplified event-triggered condition $\left|E_{i}(t)\right| \leq c_{i}\left|\delta_{i}(t)\right|$ is proposed. In order to guarantee the stability of MASs (7), the event-triggered control mechanism specifies the prior assumption that event-triggered function is built previously, and then the appropriate control protocol is derived using only local information.

Now, we will further prove that there exists at least one agents, whose positive lower bound on the interevent instants can rule out Zeno behaviour under the eventtriggered condition (16). Clearly, all the state measurement errors at initial time $t_{0}$ are zero. Define $m=\operatorname{argmax}_{i}\left|\delta_{i}(t)\right|$. Combined with $\left|E_{m}(t)\right| \leq|E(t)|$, one can obtain

$$
\frac{\left|E_{m}(t)\right|}{N\left|\delta_{m}(t)\right|} \leq \frac{|E(t)|}{|\delta(t)|}
$$

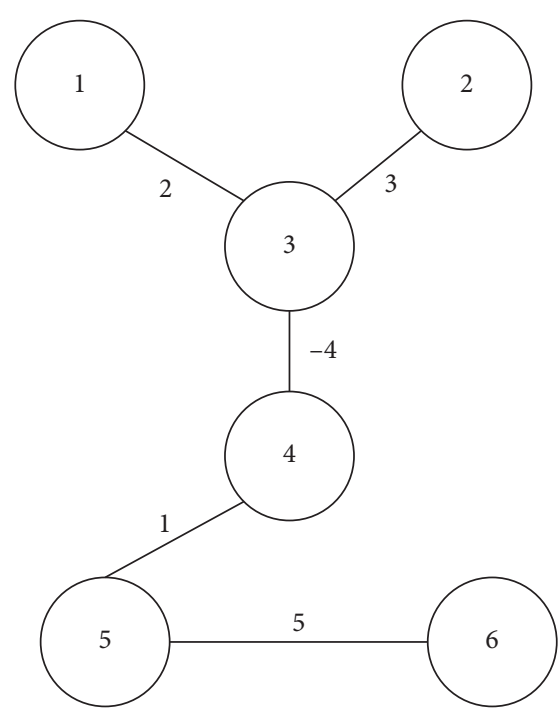

Figure 1: Communication topology.

Similar to [30], the next interevent interval of agent $m$ is bounded by $\tau_{m}$, which satisfies $\left(\left(\tau_{m}\|L\|\right) /\left(1-\tau_{m}\|L\|\right)\right)=c_{m}$ and $\tau_{m}=\left(c_{m} /\left(\left(1+c_{m}\right)\|L\|\right)\right)>0$.

Thus, under the event-triggered condition (16), a distributed finite-time bipartite consensus problem is solvable.

\section{Simulation Results}

In this section, the simulation example is presented to illustrate the effectiveness of the proposed distributed eventtriggered control strategy in finite time.

Considering a MASs with 6 agents, the communication topology is shown in Figure 1. The initial values of the MASs are randomly generated as $x(0)=\left[\begin{array}{llllll}-1 & -2 & 2 & -3 & 0 & 1\end{array}\right]^{T}$. From Figure 1, the corresponding Laplacian matrix $L$ is given by

$$
L=\left[\begin{array}{cccccc}
2 & 0 & -2 & 0 & 0 & 0 \\
0 & 3 & -3 & 0 & 0 & 0 \\
-2 & -3 & 9 & 4 & 0 & 0 \\
0 & 0 & 4 & 5 & -1 & 0 \\
0 & 0 & 0 & -1 & 6 & -5 \\
0 & 0 & 0 & 0 & -5 & 5
\end{array}\right]
$$

Here, the method to sampled data is utilized to simulate the event detection over the whole process. Then, the sampling time is set to $h=0.01$. In addition, the permitted range $\alpha$ and $\beta$ are set to 0.8 and 0.9 in real-time control, respectively.

Simulation results are shown in Figures 2 and 3 . Figure 2(a) shows the evolution of each agent's state; Figure 2(b) reveals each agent's own event sequence. From Figure 2, we calculate the average event-triggered interval overall mobile agents $h_{\text {avg }}=0.1082$. By comparison with sampling time $h=0.01$, regarding reduction in the frequency of control actions, the proposed event-triggered strategy has the advantages without degraded performance. 


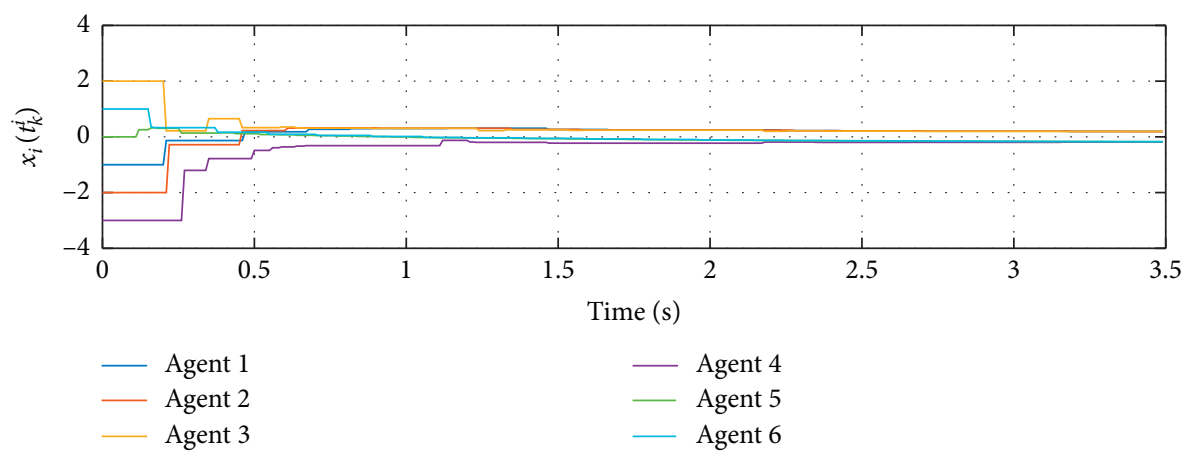

(a)

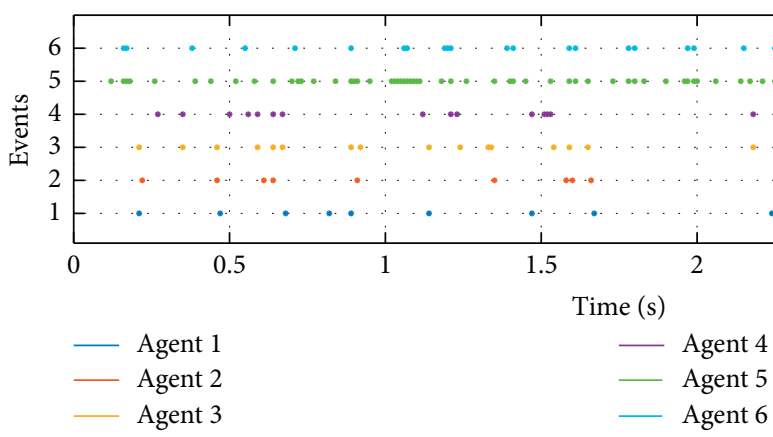

(b)

Figure 2: Simulation results of bipartite consensus for MASs. (a) The evolution of $x_{i}\left(t_{k}^{i}\right)$ for $i \in v$. (b) The event sequences of $N=6$ when $h=0.01 \mathrm{~s}$.

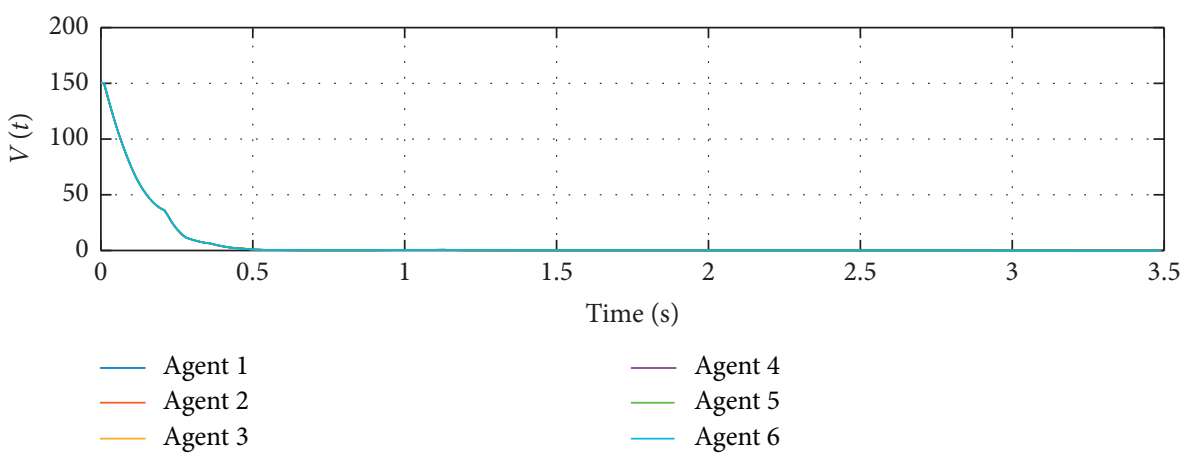

(a)

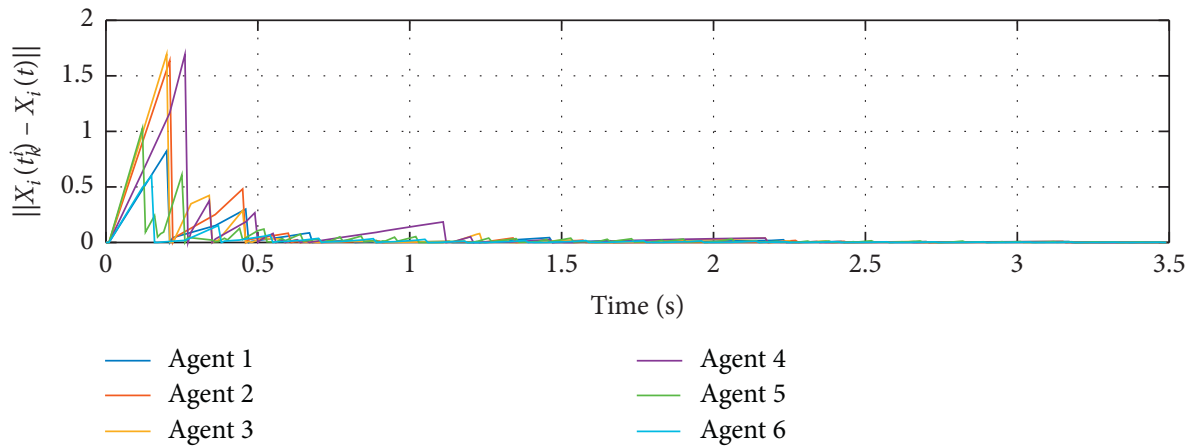

(b)

Figure 3: Simulation results of bipartite consensus for MASs. (a) The evolution of the Lyapunov function $V(t)$ in Theorem 1 for $i=1,2, \ldots, N$. (b) The evolution of $\left\|x_{i}\left(t_{k}^{i}\right)-x_{i}(t)\right\|$ for $i=1,2, \ldots, N$. 
More interestingly, it can be revealed that our event generator results in an average maximum release sampling interval of 0.1082 . Figure 3(a) presents $V(t)$ in Theorem 1 is finite-time convergent to 0; Figure $3(\mathrm{~b})$ illustrates the fluttering of the measurement error $x_{i}\left(t_{k}^{i}\right)-x_{i}(t)$. As a consequence, it indicates that the proposed finite-time coupled with the event-triggered scheme not only reduces the frequency of control actions with the better bipartite consensus performance but also achieves a bipartite consensus in finite time.

\section{Conclusion}

In this paper, we deal with the distributed finite-time bipartite consensus for MASs via event-triggered control algorithms. Combined with finite-time stability theory and event-triggered control strategy, a novelty nonlinear distributed bipartite consensus protocol is proposed over a structurally balanced signed graph. Differing from asymptotic convergence, all agents are driven to achieve a bipartite consensus by a finite-time control protocol in a settling time. Comparing with the continuous-time controller, the proposed event-triggered control scheme was proven capable of reducing the frequency of control actions. Moreover, a relationship between the convergence time and the eventtriggered condition was derived, which shows that the function threshold achieves a compromise between the control actions cost and the system performance. At last, the simulation result is presented to demonstrate the effectiveness of the theoretical results. In the future, finite-time distributed event-triggered bipartite consensus for MASs with delays in communication networks, and high-order stochastic nonlinearity will be further researched.

\section{Data Availability}

The data used to support the findings of this study are available from the corresponding author upon request.

\section{Conflicts of Interest}

The authors declare that they have no conflicts of interest.

\section{Acknowledgments}

The work was supported by the Fundamental Research Funds for the Central Universities (Grant no. 3132020140)

\section{References}

[1] G. Xu, W. Shen, and X. Wang, "Applications of wireless sensor networks in marine environment monitoring: a survey," Sensors, vol. 14, no. 9, pp. 16932-16954, 2014.

[2] S. He, M. Wang, S. L. Dai et al., "LeaderCfollower formation control of USVs with prescribed performance and collision avoidance," IEEE Transactions on Industrial Informatics, vol. 15, no. 1, pp. 572-581, 2018.

[3] C. Lin, Z. Lin, R. Zheng et al., "Distributed source localization of multi-agent systems with bearing angle measurements," IEEE Transactions on Automatic Control, vol. 61, no. 4, pp. 1105-1110, 2015.
[4] M. Shaukat and M. Chitre, "Adaptive behaviors in multiagent source localization using passive sensing," Adaptive Behavior, vol. 24, no. 6, pp. 446-463, 2016.

[5] C.-C. Hua, K. Li, and X.-P. Guan, "Semi-global/global output consensus for nonlinear multiagent systems with time delays," Automatica, vol. 103, pp. 480-489, 2019.

[6] J. Qin, W. Fu, H. Gao et al., "Distributed $k$-means algorithm and fuzzy $c$-means algorithm for sensor networks based on multiagent consensus theory," IEEE Transactions on Cybernetics, vol. 47, no. 3, pp. 772-783, 2016.

[7] C.-C. Hua, K. Li, and X.-P. Guan, "Leader-following output consensus for high-order nonlinear multiagent systems," IEEE Transactions on Automatic Control, vol. 64, no. 3, pp. 1156-1161, 2019.

[8] R. Olfati-Saber, "Flocking for multi-agent dynamic systems: algorithms and theory," IEEE Transactions on Automatic Control, vol. 51, no. 3, pp. 401-420, 2006.

[9] H. Atrianfar and M. Haeri, "Adaptive flocking control of nonlinear multi-agent systems with directed switching topologies and saturation constraints," Journal of the Franklin Institute, vol. 350, no. 6, pp. 1545-1561, 2013.

[10] Y. Shen, Z. Kong, and L. Ding, "Flocking of multi-agent system with nonlinear dynamics via distributed event-triggered control," Applied Sciences, vol. 9, no. 7, p. 1336, 2019.

[11] P. Xu, H. Zhao, G. Xie, J. Tao, and M. Xu, "Pull-based distributed event-triggered circle formation control for multiagent systems with directed topologies," Applied Sciences, vol. 9, no. 23, p. 4995, 2019.

[12] P. Xu, G. Xie, J. Tao et al., "Observer-based event-triggered circle formation control for first-and second-order multiagent systems," Complexity, vol. 2020, Article ID 4715315, 12 pages, 2020.

[13] W. Fu, J. Qin, Y. Shi et al., "Resilient consensus of discretetime complex cyber-physical networks under deception attacks," IEEE Transactions on Industrial Informatics, vol. 16, no. 7, pp. 4868-4877, 2019.

[14] C. Altafini and G. Lini, "Predictable dynamics of opinion forming for networks with antagonistic interactions," IEEE Transactions on Automatic Control, vol. 60, no. 2, pp. 342357, 2014.

[15] D. Meng, Y. Jia, and J. Du, "Finite-time consensus for multiagent systems with cooperative and antagonistic interactions," IEEE Transactions on Neural Networks and Learning Systems, vol. 27, no. 4, pp. 762-770, 2015.

[16] C. Altafini, "Achieving consensus on networks with antagonistic interactions," in Proceedings of the 2012 IEEE 51st IEEE Conference on Decision and Control (CDC), pp. 1966-1971, IEEE, Maui, HI, USA, December 2012.

[17] Y. Wu and J. Hu, "Observer-based output regulation of cooperative-competitive high-order multi-agent systems," Journal of The Franklin Institute, vol. 355, no. 10, pp. 41114130, 2018.

[18] J. Hu, Y. Wu, T. Li et al., "Consensus control of general linear multiagent systems with antagonistic interactions and communication noises," IEEE Transactions on Automatic Control, vol. 64, no. 5, pp. 2122-2127, 2018.

[19] Y. Liu and L. Cheng, "Sampled-data based mean square bipartite consensus of double-integrator multi-agent systems with measurement noises," in Proceedings of 2018 Chinese Intelligent Systems Conference, Springer, Wenzhou, China, 2018.

[20] J. Liu, Y. Yu, J. Sun, and C. Sun, “Distributed event-triggered fixed-time consensus for leader-follower multiagent systems with nonlinear dynamics and uncertain disturbances," 
International Journal of Robust and Nonlinear Control, vol. 28, no. 11, pp. 3543-3559, 2018.

[21] Q. Zhu, "Stabilization of stochastic nonlinear delay systems with exogenous disturbances and the event-triggered feedback control," IEEE Transactions on Automatic Control, vol. 64, no. 9, pp. 3764-3771, 2019.

[22] J. Li, X. Chen, and Y. Tian, "Periodic dynamic event-triggered bipartite consensus for multi-agent systems associated with signed graphs," in Proceedings of the 2019 Chinese Intelligent Systems Conference, pp. 310-321, Haikou, China, October 2019.

[23] Y. Xu, J. Wang, Y. Zhang, and Y. Xu, "Event-triggered bipartite consensus for high-order multi-agent systems with input saturation," Neurocomputing, vol. 379, pp. 284-295, 2019.

[24] J. Liu, Y. Zhang, H. Liu, Y. Yu, and C. Sun, "Robust eventtriggered control of second-order disturbed leader-follower MASs: a nonsingular finite-time consensus approach," International Journal of Robust and Nonlinear Control, vol. 29, no. 13, pp. 4298-4314, 2019.

[25] B. Hu, Z.-H. Guan, and M. Fu, "Distributed event-driven control for finite-time consensus," Automatica, vol. 103, pp. 88-95, 2019.

[26] D. Meng, Y. Jia, and J. Du, "Nonlinear finite-time bipartite consensus protocol for multi-agent systems associated with signed graphs," International Journal of Control, vol. 88, no. 10, pp. 2074-2085, 2015.

[27] L. Zhao, Y. Jia, and J. Yu, "Adaptive finite-time bipartite consensus for second-order multi-agent systems with antagonistic interactions," Systems \& Control Letters, vol. 102, pp. 22-31, 2017.

[28] S. P. Bhat and D. S. Bernstein, "Finite-time stability of continuous autonomous systems," SIAM Journal on Control and Optimization, vol. 38, no. 3, pp. 751-766, 2000.

[29] Z. Meng, W. Ren, and Z. You, "Distributed finite-time attitude containment control for multiple rigid bodies," Automatica, vol. 46, no. 12, pp. 2092-2099, 2010.

[30] D. V. Dimarogonas, E. Frazzoli, and K. H. Johansson, "Distributed event-triggered control for multi-agent systems," IEEE Transactions on Automatic Control, vol. 57, no. 5, pp. 1291-1297, 2011. 